\title{
Estudio de los hábitos lectores de los estudiantes de la Universidad Europea de Madrid
}

\author{
A survey on the reading habits of the students of the Universidad Europea de Madrid
}

\author{
Belén GARCía DELGADO \\ Universidad Europea de Madrid, Madrid, España \\ belen.garcia-delgado@uem.es
}

\begin{abstract}
Resumen
Investigación de los hábitos lectores en una muestra de ciento ochenta y un estudiantes de la Universidad Europea de Madrid. Se trató de determinar y analizar los hábitos lectores de este grupo de estudiantes, tomando siempre la lectura como una actividad de ocio, y cuantificar el tiempo dedicado a la lectura comparándolo con otras actividades: televisión, radio y demás. La metodología utilizada es la distribución de un cuestionario ajustado a las características de la muestra que puede ayudarnos a evaluar las variables que queremos obtener y su análisis mediante SPSS. El perfil del lector habitual de la Universidad Europea de Madrid es mujer y cursa estudios de Comunicación y Humanidades de primer ciclo. Lee novelas y cuentos (en español y en formato papel) una o dos veces a la semana y más a menudo en vacaciones. Normalmente ve la televisión y usa Internet para consultar el correo. No lee la prensa a diario y va a la biblioteca fundamentalmente para estudiar.
\end{abstract}

Palabras clave: Hábitos lectores. Estudiantes universitarios. Universidad Europea de Madrid. España. Ocio.

\section{Presentación}

En este artículo se van a detallar los resultados obtenidos con una encuesta sobre hábitos lectores - atendiendo únicamente a la lectura de ocio, es decir aquella que se realiza con la finalidad de ocupar el tiempo libre. Jorge Larrosa (2006), define este tipo de lectura como: "lectura de formación, como actividad que tiene que ver con la subjetividad del lector, no solo con lo que el lector sabe, sino con lo que es", como algo que nos forma, mecanismo de evasión del mundo real y del yo real; no se reduce a un medio para adquirir conocimientos en el ámbito universitario. Se ha elegido el nivel de educación superior debido a afirmaciones como la de Blanco Reinosa (2007) - "El $22 \%$ de los universitarios reconoce que no lee nunca un libro"- y de obras como la de Lapèlerie (2001), Larrosa (2006), Machado (2002) y Miguel Díaz (2006), en las que se destaca la importancia de la lectura en este tipo de estudiantes.

\begin{abstract}
The aims of this study are to determine the influence and analyze the importance of reading habits as a leisure activity, and quantify the time dedicated to it, compared with other media, as television and radio. A questionnaire was used, adapted to characteristics of the population studied, and the data was processed with SPSS. To sum up, the profile of the most usual reader in the Universidad Europea de Madrid, is a woman, attending a degree in Communication and Humanities as a freshmen or sophomore. She reads novels and tales (in paper format and in Spanish) once or twice a week and much more during the holidays. She usually watches TV and uses Internet mainly to read the mail. She does not read the press everyday and goes to the library mainly to study.
\end{abstract}

Keywords: Reading habits. Spain. Higher education students. Universidad Europea de Madrid. Leisure time.

\section{Objetivos}

No se pretende tomar como ejemplo el caso de la Universidad Europea de Madrid (UEM), sino simplemente mostrar un modelo de investigación realizado en 2008 con el fin de que se pueda aplicar en futuros análisis.

Tampoco se intenta constatar una realidad existente en la UEM, sino analizar las causas de la misma, así como los factores que favorecen o dificultan los hábitos lectores de los alumnos de la UEM.

Los objetivos planteados a los que se pretende dar respuesta con este trabajo entre otros son (Almaninos Chica, 2006; Borges Regedor, 2003; FGEE, 2007; Goldin, 2006; Miguel, 1998; Moreno Sánchez, 2000):

1. Determinar la influencia que el entorno familiar tiene en los hábitos lectores, analizando el número de libros en el hogar, para valorar la influencia en los hábitos lectores. 
2. Analizar la repercusión que tienen los medios tecnológicos en los hábitos de lectura, a través de indicadores como el número de televisiones en el hogar, conexión a Internet con o sin banda ancha, etc.

3. Cuantificar la dedicación del tiempo de ocio a la lectura, comparándolo con otras actividades como televisión, radio, etc.

4. Analizar las actividades que se realizan en Internet.

5. Analizar el idioma y soporte (digital o impreso) de la lectura.

6. Determinar los aspectos y formas que motivan a los encuestados a acceder libros, como actividad íntimamente relacionada con los hábitos lectores.

7. Determinar el papel y utilización de bibliotecas, tanto para la lectura como para otras actividades.

8. Por último, pero no por ello menos importante, a pesar de la dificultad de sistematizarlo, hemos intentado analizar los géneros y tipos y cantidad de libros que se leen o se compran.

A continuación pasamos a detallar la metodología, los resultados y conclusiones obtenidas.

\section{Metodología}

El estudio ha consistido en un análisis de diferentes variables a través de un cuestionario distribuido a una muestra de ciento ochenta y un alumnos de diferentes licenciaturas de la Universidad Europea de Madrid, lo que supone una desviación de un más, menos ocho por ciento; cifra inevitable debido a que para disminuirla habría sido preciso encuestar a un número muchísimo mayor, de por lo menos mil personas, lo que hubiera hecho inviable el estudio.

\subsection{Instrumentos}

Se ha seleccionado y diseñado la técnica de la encuesta tras el estudio de obras de carácter metodológico (Cea D'ancona, 2004; Payné, 1951; Santos Guerra, 1998). Podemos diferenciar así dos líneas fundamentales a la hora de investigar acerca de los índices de lectura de una muestra determinada:

- Directa: el comportamiento lector a través de encuestas y estudios.

- Indirecta: hábitos sociales generales de consumo o producción editorial.
Evidentemente, nosotros hemos reflexionado acerca del hábito lector a través de encuestas y el estudio de las mismas, utilizando por lo tanto una línea directa.

También nos hemos centrado en el hábito lector a través de diversos ámbitos o entornos: la lectura en bibliotecas, la lectura privada, la lectura en diversos soportes y los hábitos de compra.

En cuanto a las variables de medida de la conducta lectora diferenciamos dos tipos de fuentes:

- Directas: aspectos medibles del comportamiento lector a través del tiempo dedicado a la lectura voluntaria y el número de libros leídos en el último año.

- Indirectas, que miden el número de libros que compra al año, el número de préstamos realizados y las visitas a bibliotecas.

Nosotros hemos hecho uso de ambos tipos de fuentes, tanto directas como indirectas, por lo que según las mismas podremos determinar más fácilmente si un sujeto es un lector habitual u ocasional- o un no lector. Al hilo de esto, Gómez Soto (1999) destaca la importancia de combinar diferentes tipos de fuentes: "la necesidad de integrar y contrastar en cada encuesta varias perspectivas de análisis ya que ningún indicador es perfecto".

Pero, a pesar de todo, siempre existe un cierto sesgo ya que cada estudio del hábito lector es diferente, no se parte siempre de la misma base, así:

- No siempre el encuestador parte del mismo concepto de hábito de lectura.

- Las cuestiones que plantea la encuesta y el modo de formularlas, así como las posibles alternativas de respuesta, no son iguales en todas las ocasiones.

- Los resultados varían mucho según la interpretación subjetiva del encuestado.

También varían mucho los criterios para definir al lector según el estudio, ya que hay que tener en cuenta:

- El umbral mínimo de lectura: A veces se tiene en cuenta un libro al año, a veces tres, $y$, otras, estar leyendo un libro en el momento de realizar la encuesta.

- Frecuencia del acto lector: Si se considera una vez en los últimos tres meses, leer de vez en cuando, haber leído un libro en el último año... 
- Afición a la lectura: Depende de los distintos niveles de respuestas y de las apreciaciones del entrevistado.

De manera que, no se pueden comparar unos estudios con otros para definir de forma certera el hábito lector de una muestra determinada, ya que cada uno considera unos determinados factores y conceptos distintos.

Con el fin de elaborar un cuestionario adecuado hemos tomado como modelo varias obras de referencia sobre el tema (Almaninos Chica, 2006; Borges Regedor, 2003; Barómetro, 2007; Barómetro, 2008; Barómetro, 2010; Goldin, 2006; Miguel, 1998; Moreno Sánchez, 2000). Así se han tenido en cuenta los siguientes aspectos para la confección del mismo.

\subsection{Variables de control}

Las variables de control que han sido tratadas en nuestro cuestionario en vinculación con los hábitos lectores son:

- Edad: En nuestro caso no es algo relevante para nuestro estudio, ya que no podemos establecer ningún tipo de diferencia entre unos grupos de edad y otros porque casi todos los alumnos pertenecen al mismo rango de edad (de 19 a 25 años), existiendo solo sutiles diferencias entre ellos. Pero, podemos afirmar que los tópicos se cumplen, ya que los jóvenes universitarios ejecutan todo tipo de conductas alternativas de ocio, pero no la lectura.

- Sexo: Hay una equiparación entre ambos sexos (distribución de $40 \%$ de mujeres frente al $60 \%$ de hombres) debido a las similares posibilidades educativas. Incluso hay una ligera superioridad porcentual en el sector femenino, reflejado entre otros aspectos en el mercado editorial, con el incremento de publicaciones dirigidas a la mujer, tanto en el sector del libro como en las publicaciones periódicas. Así, al observar los resultados de esta encuesta vemos que son las mujeres las que leen con mayor frecuencia, mayor número de horas y más libros.

- Estatus: El estatus socio-económico funciona como condicionante en la proporción de lectores, en la frecuencia y tipología de la lectura. Aunque el grupo social funciona como motivador de los hábitos lectores, en unos casos potenciándolos, al producirse en el mismo contexto, o actuando como motivo percibido por el sujeto para elevar su nivel social, en nuestro caso, casi todos los usuarios pertenecen al mismo estatus socioeconómico, ya que se trata de una universi- dad privada, por lo que esta no es una variable que produzca conclusiones significativas.

\subsection{Secuencia y estructura}

Se ha pretendido mantener una secuencia lógica como es: entorno, ocupación del ocio, lectura, compra de libros y utilización de las bibliotecas; estableciendo así ocho grupos de preguntas.

1. Datos personales: La encuesta era anónima para incentivar así la confianza y evitar la identificación. Únicamente hemos incluido edad, sexo, titulación y curso.

2. Entorno: Se busca averiguar el ambiente en el que el encuestado se mueve y cómo éste puede haber influido en su caso, se realizaron preguntas referidas a los libros que existen en el hogar, medios tecnológicos como televisiones, ordenadores con conexión a Internet, grandes competidores de los libros. Abarca de las preguntas 1 a 5 del cuestionario del anexo.

3. Ocupación del ocio: Se distingue entre las distintas formas de utilización del tiempo libre como lectura, radio, televisión e Internet; siendo así la parte más importante del cuestionario (preguntas de la 6 a la 17 del cuestionario anexo).

4. Soporte y lengua de lectura: Se trata de averiguar si se trata de libros impresos o digitales (1), así como el idioma (2) en que se realiza, reflejando así cuáles son sus preferencias y aficiones (preguntas 18 a 21 del cuestionario anexo).

5. Motivación y dedicación a la lectura: Se indaga la razón que lleva a los encuestados a dedicarse a la lectura, así como el tiempo de dedicación a la misma, el momento en que se realiza y el tipo de publicación (libros o publicaciones periódicas). Ocupa las preguntas 22 a 28 del cuestionario anexo.

6. Compra de libros o publicaciones: Se pretende conocer el número de libros adquiridos, el tipo de libros, la frecuencia, los motivos y la influencia en la decisión de compra (preguntas 29 a 38 del cuestionario del anexo).

7. Utilización de bibliotecas: No cabe duda de que uno de los grandes promotores de la lectura son las bibliotecas, por lo que el uso que se haga de ellas nos da grandes pistas acerca del tipo de lector que es el encuestado (preguntas 39 a 44 del cuestionario del anexo). 
8. Aficiones literarias: Es un objetivo ambicioso sobre todo para los alumnos de carreras técnicas, pero no lo es tanto para los de Periodismo, Traducción o similar (preguntas 45 a 46 del cuestionario del anexo).

\subsection{Diseño del cuestionario}

Se ha iniciado el cuestionario con las preguntas más fáciles y no comprometidas, facilitando así el proceso a la hora de contestarlo por parte del entrevistado.

\subsection{Redacción de las preguntas}

Es uno de los aspectos esenciales, tal y como explica Payne (1951), las preguntas deben ser cortas, cerradas, simples y directas. Aunque debido a las dificultades que existen a la hora de cuantificar aspectos culturales como éste, se han llevado a cabo estas pautas en la medida de lo posible.

\subsection{Distribución del cuestionario.}

Se ha realizado en la biblioteca de la Universidad Europea de Madrid durante dos días hábiles, aunque la muestra no está afectada ya que, de forma general los alumnos se dirigen a la biblioteca a estudiar, por lo que no se puede considerar a los entrevistados como proclives a la lectura y su entorno.

Para analizar cuantitativamente los resultados obtenidos se ha utilizado el programa informático SPSS.

\section{Resultados}

Si algo hemos deducido en este estudio es que para el desarrollo de hábitos lectores, en cuanto a ocio y entretenimiento se refiere, es importante el marco político, cultural y económico de la sociedad en la que está inmerso el individuo, que afecta a la frecuencia de lectura, tipo de lectura, del material leído y a la función social que se otorga al acto lector.

Por tanto, el interés por la lectura depende del conjunto de valores, expectativas y motivaciones que genere el contexto social. Como elemento determinante individual del hábito lector figura la educación del sujeto, entendida como proceso de socialización a partir del contexto sociocultural.

\subsection{Factores a tener en cuenta al estudiar el hábito lector}

La tendencia actual en cuanto a gustos lectores se refiere tiene como objetivo la lectura fácil, fugaz, perecedera, de tipo práctico. Con esto se consigue subir el índice de lectura global, el número de lectores ocasionales, pero no el hábito lector ni la capacidad crítica, ni la independencia de pensamiento de los sujetos, ni el nivel cultural general; que son los factores que realmente importan (Cerrillo, 2002; Cerrillo, 2005).

La lectura forma parte esencial de nuestra vida cotidiana, es una herramienta imprescindible de uso; la diferencia está en: lo qué se lee, cómo se lee, cuánto se lee, por qué se lee, cuándo se lee y cómo se accede al libro.

En cuanto al primer objetivo planteado -el último en la lista anterior ( $h$ ): qué se lee-, podemos afirmar que en nuestra muestra es la literatura lo que más se lee (52\%), sobre todo por las mujeres en cuanto a sexo se refiere, y en cuanto a tipo de titulación, los del área de Comunicación y Humanidades (56\%). Pero la materia de literatura engloba diversos géneros, de entre los cuales destacan la novela y el cuento (93\%). Según el tipo de novelas, encontramos que el sector femenino prefiere las novelas de intriga $(26 \%)$ y el masculino las de aventuras $(27 \%)$.

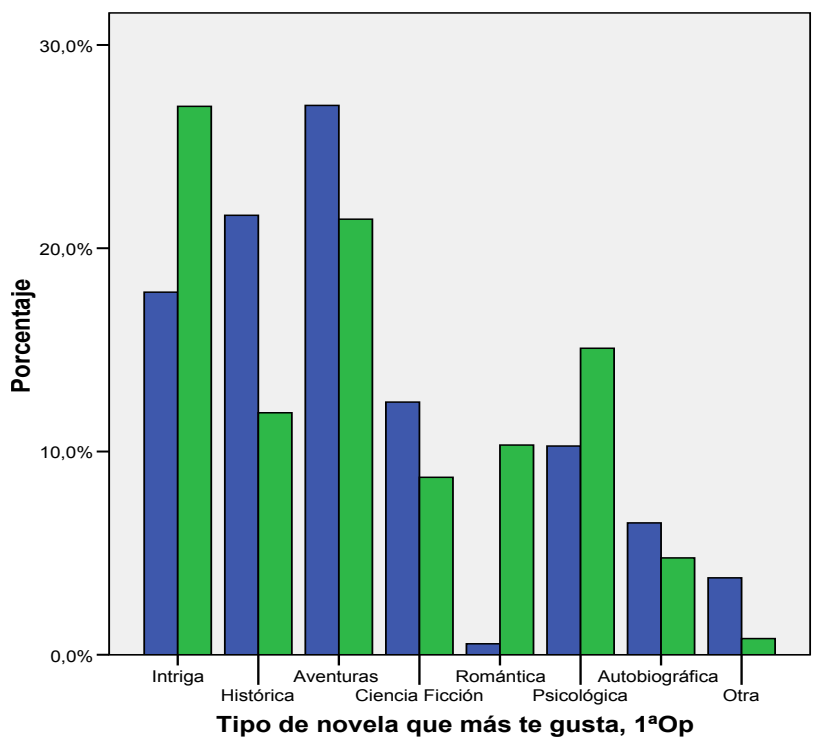

Figura 1. Tipo de novela que se prefiere por sexo

Si nos referimos a la prensa diaria, son las mujeres las que más la leen (44\%); y según el tipo de titulación, es el área de Comunicación y Humanidades $(58 \%)$ obviamente, el que más lee este tipo de publicación, necesaria para el desarrollo de su carrera.

Siguiendo la línea del material no librario, en las publicaciones periódicas, las féminas se decantan por las revistas $(29 \%)$, mientras que los hombres prefieren la prensa deportiva $(30 \%)$. Cosa por otra parte evidente, ya que general- 
mente cada tipo de publicación se elabora expresamente para un sexo determinado; siendo las revistas expresamente creadas para las chicas y la prensa deportiva para los chicos.

Para el objetivo acerca de soportes e idioma, hay varios aspectos a tener en cuenta:

- Soporte: a pesar del gran impacto de las nuevas tecnologías, los libros se leen en soporte papel (85\%), no en formato digital.

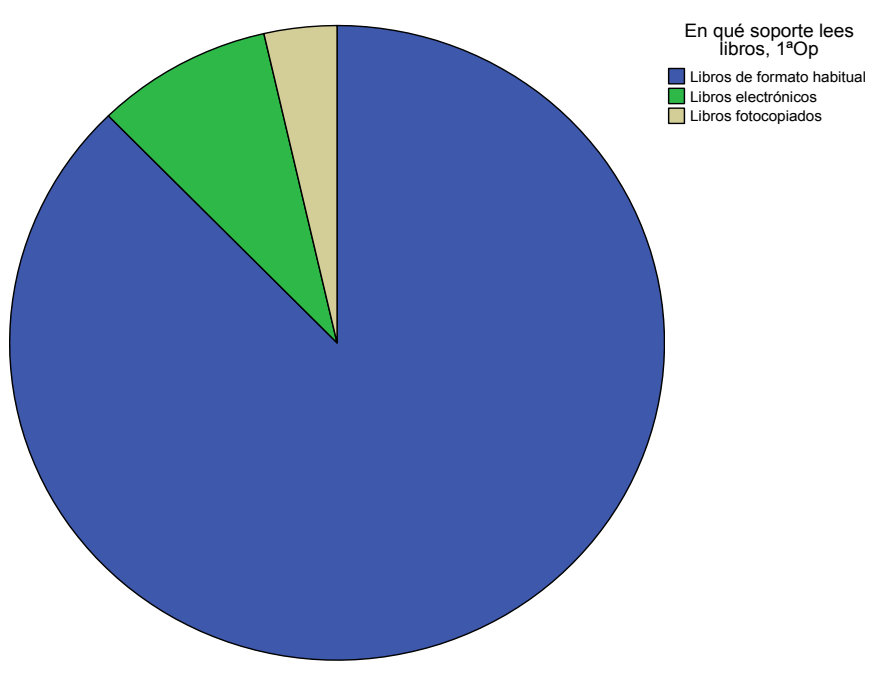

Figura 2. Soportes empleados para la lectura: impreso, electrónico o fotocopiado.

- Lengua: observamos que los jóvenes universitarios no se esfuerzan en leer en otras lenguas, sino que leen en español principalmente $(93,2 \%)$. El segundo idioma de lectura es el inglés $(1,1 \%)$, lengua en la que leen una mayoría de mujeres $(49,4 \%)$ y un $50 \%$ de alumnos del área de Ciencias de la Salud, suponemos que debido a la necesidad que en este campo se tiene de leer publicaciones científicas, y también los de Comunicación.

La tercera pregunta expuesta cuánto se lee, hace alusión a la cantidad de libros de ocio leídos.

Observamos así que el $40 \%$ de los encuestados lee todos o casi todos los días, siendo las mujeres las que leen con mayor frecuencia y mayor número de horas.

Comunicación y Humanidades es el área temática que mayor tiempo emplea en esta actividad y que lee el más alto número de libros.

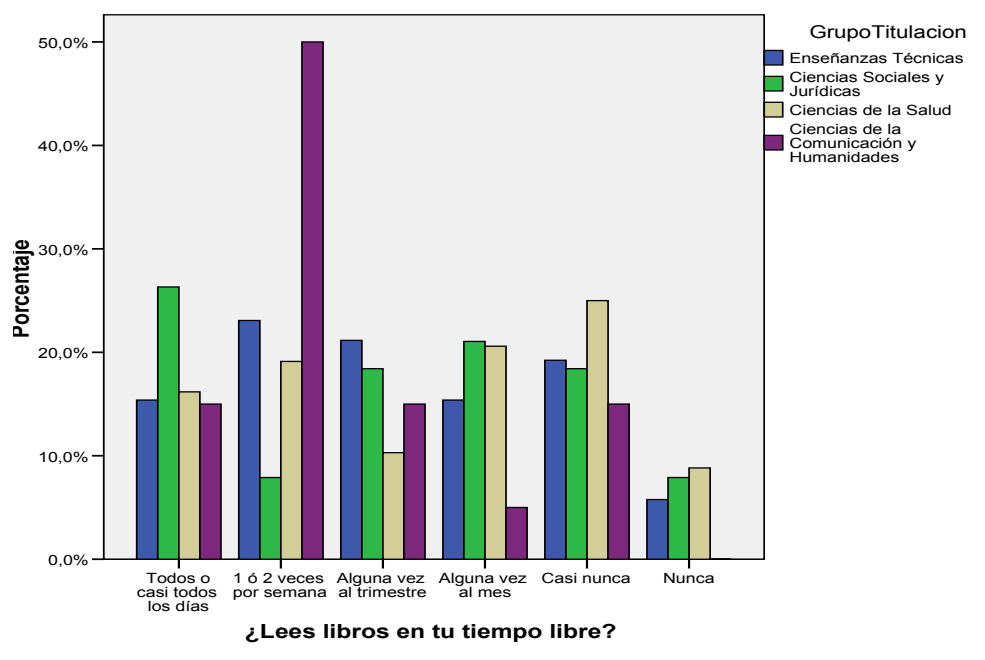

Figura 3. Frecuencia con la que se lee en el tiempo libre por titulaciones.

Otro factor que implica un alto índice de lectura es el número de libros comprados, así en este estudio la media de libros comprados es 4,47 al año, destacando como es habitual el área de Comunicación y Humanidades $(60 \%)$ como el sector más comprador y siendo Ciencias de la Salud los que menos leen, sin embargo las diferencias entre unas titulaciones y otras no son muy significativas a pesar de la mayor propensión teórica de las carreras de letras.

Un concepto muy importante a tener en cuenta es el por qué se lee -lo que responde al objetivo f-. Así el 53,6\% de los encuestados afirma leer por entretenimiento y un $59,4 \%$ dice no practicar este hábito más a menudo por falta de tiempo. Esta afirmación la realiza una mayoría de mujeres.

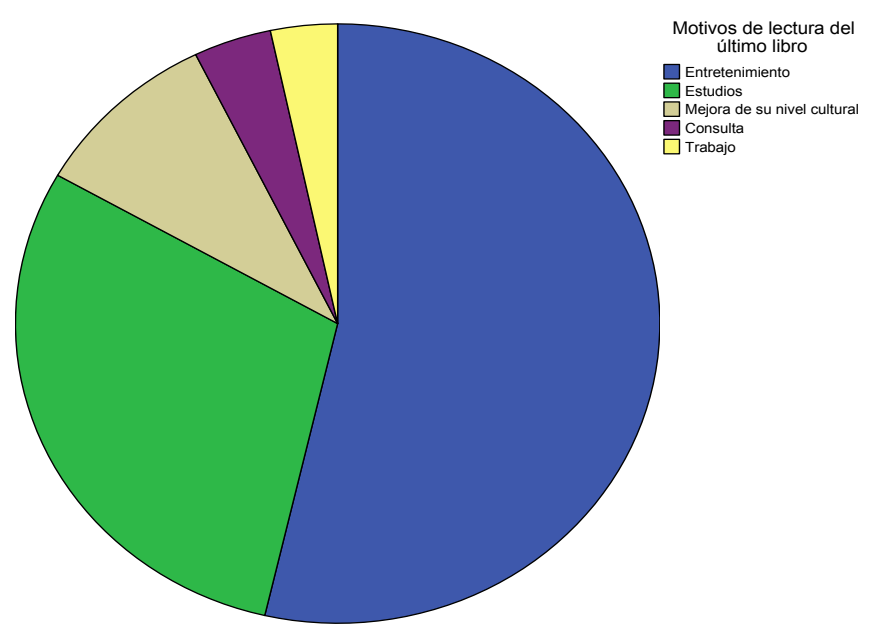

Figura 4. Motivos por los que se lee 
A la hora de adquirir libros, es el estudio el principal motivo de compra elegido por los estudiantes del área de Ciencias de la Salud (con un $53 \%$ ) y no el de ocio o entretenimiento, como era de esperar.

Lo que mueve a los encuestados a comprar libros es fundamentalmente el consejo de amigos o conocidos, con un $36,2 \%$, más que las críticas realizadas en periódicos...

También se debe tener en cuenta cuándo se lee: en qué período del año y si leen ahora más o menos que antes. En este estudio hemos observado que un $52 \%$ lee más en períodos vacacionales que durante el resto del año y un $46,9 \%$ dice que ahora lee menos que antes, realizando esta afirmación un $57,9 \%$ de estudiantes del área de Comunicación y Humanidades, lo cual significa que la dedicación al estudio de los estudiantes se contrapone a la lectura de ocio. Si vamos más allá en cuanto a cuándo se lee en la vida de una persona, podemos ver que es durante el período de formación escolar cuando se debe fomentar y promover la lectura para crear un hábito en la persona.

La forma de acceder al libro entre los estudiantes de la Universidad Europea de Madrid es en un $44,7 \%$ la compra, sobre todo en el sector femenino $(47,2 \%)$ y en el ámbito de Comunicación y Humanidades (60\%). Cuando se trata de comprar, son los libros de texto los preferidos por un $40,4 \%$, siendo de los alumnos de Enseñanzas técnicas los que adquieren más libros de ocio (con un $18 \%$ ).

Las otras formas de acceso al libro de ocio -y por tanto a la lectura, son las bibliotecas - a las que acuden una mayoría de chicas que estudian carreras del ámbito de Comunicación y Humanidades $(76,7 \%)$. De este mismo perfil son los encuestados que están inscritos en estas unidades de información.

Pero no existe correlación alguna entre la frecuencia de visita a las bibliotecas y la práctica del hábito lector -objetivo g-, ya que la actividad que más realizan aquí los estudiantes de esta universidad es el estudio por encima de cualquier otra, como ámbito tranquilo que invita al estudio. El servicio de préstamo lo usa un $51,4 \%$ de mujeres, del área de Comunicación y Humanidades $(26,2 \%)$ principalmente.

\subsection{La lectura en los medios de comunicación}

En los últimos veinte años se han universalizado los medios de comunicación audiovisuales, adquiriendo una posición de predominio absoluto sobre cualquier otra alternativa. Por lo que el ocio se ha transformado en un acto de recep- ción de estímulos fugaces en busca del disfrute y placer (Cordón, 2006).

Sin embargo, al realizar esta encuesta hemos comprobado que unos medios de comunicación se utilizan más que otros -lo cual responde al objetivo c-, por ejemplo la radio apenas se escucha, y si se hace, tiene lugar a la par que otras tareas como la conducción o el estudio. Por el contrario, se dedica mucho tiempo (entre 120 y 180 minutos diarios) a ver la televisión, sobre todo en el caso de los hombres. Pero en ninguno de los casos —ni en el de la radio ni en el de la televisión- los encuestados han manifestado aprovecharse de los mismos con el fin de informarse de programas acerca de libros.

El tercer medio de comunicación destacado es Internet, que se utiliza mucho para la realización de diversas tareas como - respondiendo así al objetivo d- buscar información, ver el correo, chatear a través de las redes sociales, pero no para leer libros, consultar críticas sobre los mismos, ni tampoco para comprarlos a través de librerías en línea. Podemos afirmar por tanto, que a pesar de que Internet es una herramienta tremendamente utilizada, aún a día de hoy existen ciertas dificultades a la hora de leer documentos en pantalla, o realizar compras en línea de libros, sin tener éstos en la mano. Se sigue percibiendo así el libro como algo tangible, que se puede tocar y no como algo etéreo.

Concluimos de esta manera, que a pesar de que los medios de comunicación podrían ser un paso previo a la lectura, sirviendo como enlace o acceso a esta actividad, la realidad es que los encuestados no los utilizan para esta actividad.

\section{Conclusiones: perfil del lector de la Universidad Europea de Madrid, la constatación de la realidad}

En nuestro caso, resulta evidente que los tópicos se cumplen, es decir que cada vez se lee menos, y lo que es peor, no hay grandes expectativas de cambio al respecto. Sin embargo, el Espacio Europeo de Educación Superior, conocido como Bolonia, propone ciertas novedades desde el punto de vista educativo, que podrían beneficiar en gran medida el hábito lector. Éstas son entre otras, el nuevo concepto de aprendizaje donde el centro de atención es el alumno, otorgándole tareas como la creación de sus propios apuntes, lo que implica que lea manuales, utilice diferentes fuentes de información, etc. Todo esto conlleva que descodifique información, que practique la actividad lectora; por lo que supondría un gran beneficio para la enseñanza y, lo que es más, fomentaría el futuro hábito lector. 
Pero, la realidad es que mucho tiene que cambiar la situación, ya que actualmente los alumnos se limitan a tomar apuntes, sin leer o consultar las distintas fuentes recomendadas, sin informarse diariamente de las noticias actuales con la lectura de periódicos en distintos soportes, acuden a la biblioteca exclusivamente a estudiar, hacen uso de Internet solo para consultar el correo electrónico y chatear y, por último pero no por ello menos importante; eligen otras alternativas de ocio, que difieren de la actividad lectora.

Así el perfil del lector más habitual del estudiante de la Universidad Europea de Madrid, es mujer, cursa estudios de primer ciclo de una titulación del área de Comunicación y Humanidades, lee novelas y cuentos (en soporte papel y en lengua española) una o dos veces por semana y con mayor asiduidad durante las vacaciones, ve habitualmente la televisión, utiliza Internet para consultar fundamentalmente el correo, no lee la prensa a diario y acude a la biblioteca fundamentalmente para estudiar.

Hemos respondido así a todos los objetivos planteados, con excepción de los dos primeros - a y $b$-, ya que resulta muy complicado comprobar la relación que existe entre el equipamiento del que se dispone en el hogar (número de libros, televisiones, conexión a internet, etc.) con respecto a los hábitos lectores. No hemos observado una clara correlación entre ambos conceptos que nos permita sacar conclusiones, ni siquiera indicios.

\section{Notas}

(1) Tenemos que tener en cuenta que aunque a día de hoy la lectura electrónica se está extendiendo a través del auge de los dispositivos electrónicos, la encuesta se realizó en 2008 cuando aún estos dispositivos eran muy caros y, por tanto, poco utilizados. Los datos del Barómetro de hábitos de lectura y compra de libros del citado año ni siquiera mencionan el porcentaje de lectores que leen a través de ereaders, centrándose principalmente en el uso que se hace de internet con fines literarios. Ya en 2010 si se aporta el dato, siendo de tan solo de un $1 \%$.

(2) Los estudiantes universitarios han estudiado en su mayoría inglés (Contreras, 2008), o de forma excepcional otras lenguas, por lo que las aficiones particulares de algunos jóvenes pueden ser un estímulo para el uso del inglés. Por ejemplo la visualización de películas en inglés y la lectura de los subtítulos, en castellano o en inglés, y, por supuesto en este caso, la lectura de libros de ocio en lengua original.

\section{Referencias}

Alaminos Chica, A. (2006). Elaboración, análisis e interpretación de encuestas, cuestionarios y escalas de opinión. Alcoy: Marfil.
Blanco Reinosa, Y. (2007). El 22\% de los universitarios reconoce que no lee nunca un libro. // El Mundo. 28/11/2007, 1-2.

Borges Regedor, A. (2003). As práticas de compra e de lectura voluntária en lectores de biblioteca pública. Salamanca: Universidad.

Cea D'ancona, M. A. (2004). Métodos de encuesta: teoría y práctica, errores y mejora. Madrid: Síntesis.

Cerrillo Torremocha, P. (2002). Libros, lectores y mediadores. Cuenca: Ediciones de la Universidad de Castilla-La Mancha.

Cerrillo Torremocha, P. (2005). Lectura y sociedad del conocimiento. // Revista de Educación. Núm. extraordinario. 53-61.

Cordón García, J. A. (2006). Lecturas sobre la lectura. // Gonzalo García, C.; Hernúñez, P. Corcillum: estudios de traducción, lingüística y filología dedicados a Valentín García Yebra. Madrid: Arco/Libros. 735-763.

Barómetro de hábitos de lectura y compra de libros en 2007. Madrid: Federación del Gremio de editores de España, 2007. http://www.federacioneditores.org/0 Resources/ Documentos/Indice_Lectura_Esp_2007.pdf (2011-5-10).

Barómetro de hábitos de lectura y compra de libros en 2008. Madrid: Federación del Gremio de editores de España, 2008. http://www.federacioneditores.org/0 Resources/ Documentos/Indice_Lectura_Esp_2008.pdf (2011-5-10).

Barómetro de hábitos de lectura y compra de libros en 2010. Madrid: Federación del Gremio de editores de España, 2008. http://www.federacioneditores.org/0 Resources/ Documentos/Indice_Lectura_Esp_2008.pdf (2011-5-10).

Contreras, J. (2008) Leer en tiempos modernos: adolescentes y jóvenes profesionales frente a la lectura. // Millán, J. A. La lectura en España: informe 2008: leer para aprender. Madrid: Fundación Germán Sánchez Ruipérez, Federación de Gremios de Editores de España, 2008. 153-187.

Goldin, D. (2006). Encuesta Nacional de Lectura. Informes y evaluaciones. México: CONACULTA.

Gómez Soto, I. (1999). Mito y realidad de la lectura. Madrid: Endimión.

Lapèlerie, F. (2001). Pédagogie et lecture(s) à l'université. // BBF. 46:2, 56-65.

Larrosa, J. (2006). ¿Y tú qué piensas?: experiencia y aprendizaje". // Revista de Educación y Pedagogía. (SeptDic.) 46-57.

Machado, A. Ma (2002). Lectura, escuela y creación literaria. Madrid: Anaya.

Miguel, A. (1998). Los españoles y los libros: hábitos y actitudes hacia el libro y la lectura. Madrid: CEGAL.

Miguel Díaz, M. (2006). Metodologías de enseñanza y aprendizaje para el desarrollo de competencias: orientaciones para el profesorado universitario ante el Espacio Europeo de Educación Superior. Madrid: Alianza.

Moreno Sánchez, E. (2000). Evaluación de los hábitos de la juventud malagueña: estudio de un caso. Málaga: Ediciones de la Diputación Provincial de Málaga.

Payne, S. (1951). The Art of Asking Questions. Princeton: Princeton University Press.

Proust, M. (1989). Sobre la lectura. Valencia: Pre-textos.

Santos Guerra, M. A. (1998). Evaluar es comprender. Buenos Aires: Magisterio del Río de la Plata.

Steiner, G. (1990). Lecturas, obsesiones y otros ensayos. Madrid: Alianza 


\section{Apéndice I: Cuestionario sobre hábitos de lectura}

\section{Datos personales}

Edad:

Titulación:

Sexo:

Curso:

\section{Entorno}

1. Aproximadamente ¿cuántos libros tiene en su hogar sin considerar los libros de texto?

20 o menos

de 20 a 100

más de 100

2. ¿Cuántas televisiones tiene en su casa? 1

2 ó 3

más de 3

3 ¿Tiene ordenador(es) personal(es)? Sí / No

4. ¿Tiene conexión a Internet? Sí / No

5. ¿Con banda ancha? Sí / No

\section{Ocupación del ocio}

\subsection{Lectura}

6. ¿Suele usted leer libros en su tiempo libre? Todos o casi todos los días

1 ó 2 veces por semana

Alguna vez al trimestre

Alguna vez al mes

Casi nunca

Nunca

7. Aproximadamente ¿cuántos libros lee al año?

8. En la actualidad, ¿cuántas horas, de media, dedica diariamente a leer libros?

Menos de 1 hora

1 ó 2 horas

3 a 5 horas

6 y más horas

9. ¿Cuál es la materia del último libro leído?

Literatura

Ciencias sociales y humanidades

Libros prácticos

Científico

Técnico

Otras:

10. ¿De qué género es el último libro leído de literatura?

Ensayo

Poesía

Teatro

Novela y cuento

11. ¿Cuáles son los dos tipos de novela que más le gustan? Intriga

Histórica

Aventuras

Ciencia Ficción

Romántica

Psicológica

Autobiográfica

Otra
3.2. Radio

12. ¿Cuánto tiempo dedica al día a escuchar la radio?

13. ¿Escucha usted en la radio algún programa relacionado con los libros? Sí / No

3.3. Televisión

14. ¿Cuánto tiempo dedica al día a ver la televisión?

15. ¿Ve usted en la TV algún programa relacionado con los libros? Sí / No

3.4. Internet

16. ¿Utiliza Internet habitualmente? Sí / No

17. ¿Qué actividades realiza en Internet?

Búsqueda de información

Correo electrónico

Descarga de archivos

Búsqueda de información sobre libros

Chatear

Servicios on line (banca, reservas, compras)

Lectura de prensa

Otras

\section{Soporte y lengua de lectura}

18. ¿En qué tipo de soporte lee libros?

Libros de formato habitual

Libros electrónicos

Libros fotocopiados

19. ¿Cuál es su idioma habitual de lectura?

20. ¿Lee en otras lenguas? ¿Cuáles?

21. ¿En qué lengua está escrito el último libro leído?

5. Motivación y dedicación a la lectura

5.1. Libros

22. ¿Por cuál de los siguientes motivos ha leído el último libro?

Entretenimiento

Estudios

Mejora de su nivel cultural

Consulta

Trabajo

23. ¿Cuándo piensa en lo que leía antes y lo que lee ahora piensa que lee...?

Más que antes

Menos que antes

Igual que antes

24. En los períodos vacacionales ¿lee más, igual o menos libros que en el resto del año?

Más

Menos

Igual

25. ¿A qué se debe que no lea usted con mayor frecuencia? Le falta tiempo

Prefiere emplear su tiempo en otros entretenimientos, No le gusta leer Otros

26. ¿Cómo llegó a sus manos el último libro leído?

Compra

Préstamo

Regalo

Estaba en casa

Biblioteca 
5.2. Otras publicaciones

27. ¿Lee publicaciones periódicas?

Prensa diaria

Prensa deportiva

Revistas

Soporte electrónico

Nada

28. ¿Con qué frecuencia lee publicaciones periódicas?

5 a 7 días a la semana

3 a 4 días a la semana

1 a 2 días a la semana

Alguna vez al mes

Menor frecuencia

\section{Compra de libros o publicaciones}

29. ¿Ha comprado al menos un libro en el último año? Sí / No

30. ¿Qué tipo de libros compra?

Libro de texto

Libro no de texto Ambos

31. ¿Cuánto tiempo hace que compró el último libro? Una semana o menos

2 ó 3 semanas

Un mes

Varios meses

Un año

32. ¿Por cuál de los siguientes motivos ha hecho su última compra?

Para regalar

Estudios

Consulta

Trabajo

Ocio y entretenimiento

33. ¿Cuál es la materia del último libro comprado?

Literatura

Ciencias sociales y Humanidades

Libros prácticos

Científico

Técnico

Otra

34. De los libros de literatura ¿cuál es el género del último libro comprado?

Ensayo

Poesía

Teatro

Novela y cuento

35. En general, ¿dónde obtiene la referencia para decidir la compra de libros?

Consejo de amigos/conocidos

Librerías

Impulso

Reseñas

Catálogos

Prescripción del profesor

Otros libros

Radio/tv

Internet

Bibliotecas

36. ¿Cuántos libros ha comprado en el último año?

37. ¿Compra a través de Internet? Sí No

38. ¿Ha comprado algún fascículo en el último año? Sí / No

\section{Utilización de bibliotecas}

39. ¿En el último año ha ido usted a alguna biblioteca? Sí / No

40. ¿A qué tipo de biblioteca?

41. ¿Está inscrito en alguna? Sí No
42. ¿Con qué frecuencia visita usted la biblioteca? Todos o casi todos los días

1 ó 2 veces por semana

Alguna vez al mes

Con menos frecuencia

43. ¿Qué actividades realiza en la biblioteca?

Préstamo

Consulta

Estudiar

Uso de internet

Lectura prensa/revistas

44. ¿Ha tomado libros prestados en la biblioteca en el último mes? Sí / No

\section{Aficiones literarias}

45. ¿Podría decirme si a usted le gusta escribir? Sí / No

46. ¿Qué le gusta escribir?

Cuentos/relatos

Poesía

Diarios

Artículos/comentarios

Novelas

Otros 
